\title{
Assembly Accuracy Prediction And Optimization of Aeroengine Rotor Under The Separation Condition Of Assembly And Measurement
}

\author{
Minghua Li (D648145092@qq.com ) \\ Dalian University of Technology
}

\section{Yunlong Wang}

Dalian University of Technology

Qingchao Sun

Dalian University of Technology

\section{Xiaokai Mu}

Dalian University of Technology

\section{Research Article}

Keywords: separation condition of assembly and measurement, the datum deviation, datum independent matrix, assembly accuracy prediction and optimization

Posted Date: July 12th, 2021

DOl: https://doi.org/10.21203/rs.3.rs-680562/v1

License: (9) This work is licensed under a Creative Commons Attribution 4.0 International License. Read Full License 


\title{
Assembly accuracy prediction and optimization of aeroengine rotor under the separation condition of assembly and measurement
}

\author{
Minghua Li \\ School of Mechanical Engineering, Dalian University of Technology \\ No. 2, Linggong Road, Ganjingzi District, Dalian, Liaoning Province, China \\ e-mail: 648145092@qq.com
}

\author{
Yunlong Wang \\ School of Mechanical Engineering, Dalian University of Technology \\ No. 2, Linggong Road, Ganjingzi District, Dalian, Liaoning Province, China \\ e-mail:287254176@qq.com
}

\author{
Qingchao Sun \\ School of Mechanical Engineering, Dalian University of Technology \\ No. 2, Linggong Road, Ganjingzi District, Dalian, Liaoning Province, China \\ e-mail: qingchao@dlut.edu.cn
}

\author{
Xiaokai Mu \\ School of Mechanical Engineering, Dalian University of Technology \\ No. 2, Linggong Road, Ganjingzi District, Dalian, Liaoning Province, China \\ e-mail:muxiaokai@dlut.edu.cn
}

\begin{abstract}
The measurement and assembly of aeroengine rotor are separated from each other, the uncertainty of the test position leads to the test data cannot reflect the geometric characteristics of the rotor itself, which makes it difficult to accurately measure and predict the assembly accuracy. Combined with the fact that the
\end{abstract}


geometric characteristics of parts / components are not related to the measurement datum, an assembly accuracy test and prediction method is proposed to reduce the datum deviation and ensure the consistency of test data. Firstly, the small displacement torsor is used to describe the datum deviation, the inverse matrix transformation is applied to reduce the datum deviation, and the datum independent matrix is utilized to express the pose characteristics of the parts / components, which provides the data basis for the accurate prediction of assembly accuracy. Then, the pose transfer model based on the datum independent matrix is established, which is more comprehensive and clearer than the traditional accuracy prediction model. Furthermore, a direct optimization method is also established, which is more efficient than the traditional genetic algorithm. The assembly experiment of aeroengine rotor shows that the model and method proposed in this paper are beneficial to reduce the coaxiality of the front and rear fulcrum and they can better reflect the geometric characteristics of the rotor itself. The related research also has reference significance for other large-scale and high-precision mechanical product assembly.

Keywords: separation condition of assembly and measurement, the datum deviation, datum independent matrix, assembly accuracy prediction and optimization

\section{Quick browse and highlight}

Aeroengine rotors are generally composed of multi-stage discs / drums parts, and are produced using graded manufacturing and stacking assembly technologies. The coaxiality of multi-stage rotors is often used as an important factor in evaluating its assembly accuracy. Determined by the structural characteristics of aeroengine rotors, precision turntables are used to measure the shape errors of rotors at all levels, but special hydraulic tooling is used for assembly, which makes the assembly process and the test process separate. Repeated testing and installation make it difficult for the test data to truly reflect the geometric characteristics of the rotor itself, and the precise evaluation of the coaxiality is also affected, which in turn affects the assembly accuracy of the multi-stage rotor. In this paper, by measuring the runout of the assembly contact surface of the rotor at all levels, combining the actual engineering conditions, based on the least square fitting principle and the SDT theory, an error reduction matrix is established to eliminate the evaluation of the actual center position of the rotor due to the eccentricity of the rotor placement and manufacturing errors. In addition, the datum independent matrix is used to accurately describe the position transformation of the actual 
center of the rotor at each level, thereby improving the test reliability of the multi-level rotor coaxiality, and the stacking model based on the datum independent matrix and assembly phase optimization is established to realize the assembly prediction accuracy improved of multi-level rotor, at the same time, a more efficient assembly phase calculation method is given compared with genetic algorithm.

The novelty of this paper can be described in detail based on the following formula. $T_{0-n}$ describes the overall feature transformation from bottom to top when n-stage rotor is assembled. Firstly, the error reduction matrix $T_{E-0}^{-1}$ proposed in this paper is used to eliminate the reference error, so as to ensure that the test data can truly reflect the geometric characteristics of the rotor itself; Secondly, the rotation matrix with assembly phase is introduced to transform the calculation formula of coaxiality into trigonometric function with this variable, which makes it possible to solve the problem directly (the amount of calculation is $2 \mathrm{n}$ ) and avoids the exhaustive optimization in genetic algorithm (the amount of calculation is $36^{n-1}$ ); Combined with the previous two steps, the multi-stage rotor test and stack assembly experiment are carried out, and it is found that the optimization of assembly accuracy and the improvement of calculation efficiency can be achieved.

$$
\begin{gathered}
T_{0-n}=T_{0-1} * T_{1-1} * T_{1^{\prime}-2} * \ldots \ldots * T_{m-m^{\prime}} * T_{m^{\prime}-n} \\
T_{0-1}=T_{E-0}^{-1} * T_{E-1}
\end{gathered}
$$

\section{Introduction}

Aeroengine rotors are mainly produced through graded manufacturing and stacked assembly techniques, as Fig. 1, owing to the assembly requirements of high precision and reliability, it is necessary to test each level of assembly components to verify whether the assembly error is within the required range. It is usually important to measure the runout of the assembly contact surface of the rotor at all levels as Fig. 2, and the coaxiality of the entire rotor, based on the required measurement elements, a precision turntable is generally used for related measurements. However, due to the lack of strength and stiffness of turntable for aeroengine rotor measurement and the limitation of special hydraulic tooling, the assembly process is separated from the test process, that is, the assembly process is carried out on the hydraulic tooling, while the test is carried out on the turntable. This leads to inconsistencies between the test data and the geometric characteristics of the rotor itself, the test error of each stage rotor is continuously transferred and amplified in the stacking process, which makes the assembly prediction accuracy of multi-stage rotor deviate. 
Due to the complexity and importance of aeroengine rotor assembly, it has always been one of the key tasks of aeroengine development. Different researchers have carried out detailed research on manufacturing error characterization, assembly error transmission, assembly tolerance distribution, and predictive model establishment. Zhu introduced the concept of aeroengine assembly dimension chain and the characteristics of the closed loop, combined with the aeroengine structure and assembly characteristics, proposed three typical tolerance design analysis methods suitable for aeroengines [1]. In order to realize the optimization of the tolerance distribution of thin-walled parts assembly, Chen proposed a tolerance optimization method for manufacturing and deformation [2]. Whitney proposed a tolerance expression based on the principle of homogeneous matrix transformation. This expression can describe the theoretical value of the component caused by geometric deviation and the relationship between machining error value [3]. Cai illustrated a mathematical model of the shape tolerance of cylindrical workpieces based on the mathematical definition, which explained the definition of shape tolerance more clearly and accurately [4]. Liu proposed the dualobjective optimization principle for rotor misalignment and unbalance, used Monte Carlo method to simulate the random assembly process, single-objective assembly optimization process, and dual-objective assembly optimization process to improve the rotor assembly quality [5]. Ju has established a multi-stage disc rotor structure assembly process based on rotor runout and initial unbalance optimization, which has strong engineering guiding significance for aeroengine assembly optimization, decomposition inspection, and connection surface quality evaluation [6]. Sun has studied the influence of real contact stiffness and spigot interference on the offset of the stack center, and established a finite element model of the rotor stack to further improve the prediction accuracy [7]. Rao established a tolerance allocation method based on the equal interval analysis theory, which is used to find the optimal tolerance of all dimensions and the optimal gap between all contacts in the assembly [8]. Ding proposed a state space model based on mathematical expressions to describe the assembly process of multi-level components, and through analysis showed that there is an optimal control strategy to control the variable transfer in the assembly process [9]. Thimm illustrated an optimization method based on the benchmark hierarchy tree theory to control tolerance stacking, which considered the maximum tolerance stacking and the minimum benchmark [10]. Zheng proposed a reasonable tolerance allocation strategy based on the joint game theory, which found a balance point for each assembly component to weigh the assembly cost and assembly quality [11]. For rotationally 
symmetrical aero-engine components, to control the tolerance transfer process in the assembly process, Klocke proposed a set of intelligent assembly system, which can realize the automatic distribution of disklike components with the center as the installation reference and meet certain tolerance requirements [12-14]. Yang proposed a method for optimizing assembly stacking by controlling the direction of rotation of components, and in-depth study of the assembly results of vertical assembly strategies under different measurement accuracy and measurement noise levels. This method also considers the complexity and randomness of the processing and measurement processes [15-18]. Meng considered the tolerance transfer model of the circumferential installation angle, established the relationship between coaxiality, tolerance and installation angle, and used Monte Carlo simulation to verify the assembly qualification rate, which improved the assembly reliability [19]. Shan considered the unique assembly method of the aero-engine rotor, established a dimensional chain model including dimensional deviation and position deviation, and introduced the Pearson distribution family method to make the dimensional chain model when the input deviation is non-normally distributed, it also can calculate the distribution pattern of assembly deviation [20]. Ai focuses on several aspects such as rotor misalignment control and rotor unbalance control, summarizes the control method of aero-engine vibration [21]. Ding and Jin conducted a detailed analysis of the aeroengine rotor assembly process based on the Jacobian torsor theory, and established an assembly deviation transfer chain and a multi-stage rotor assembly model [22-24]. Chen analyzed the four main methods of threedimensional tolerance analysis in detail, analyzed their main advantages, disadvantages and applications, and looked forward to the future research of three-dimensional tolerance analysis [25]. Chen proposed a calculation model of the assembly success rate based on the improved Taguchi method, and combine the Pearson distribution theory to establish a calculation model of the aero-engine assembly success rate [26,27]. Sun studied the aero engine assembly technology driven by digital twins, discussed its composition, function, and process, and analyzed in detail the assembly process control, parts selection, assembly operation guidance, assembly gap control, assembly technology status control, and assembly test data association analysis, etc. technology [28]. And the small displacement torsor is also proposed to describe the tiny poses in space [29-31]. Their work has promoted the development and application of aeroengine rotor assembly technology. The problem still need to be solved is that: owing to the rotor is usually produced in small batch, the machining deviation between parts is often different which result in the statistical results are not reliable, 
hence the representation of rotor machining error cannot simply give the expressions in the form of probability distribution; The eccentricity and tilt of rotor cannot be avoided owing to the processing conditions and placement deviation, which result in the data obtained in each test process is inconsistent and it is difficult to reflect the geometric characteristics of the rotor itself, consequently, it is necessary to reduce the inconsistency of each measurement by some methods; The datum error caused by the non-coincidence of measurement datum and coaxiality evaluation standard must be considered here, so the comprehensive transformation matrix of rotor assembly characteristics is not a simple multiplication step by step, in addition, when the rotor is assembled in the actual production line, its calculation efficiency and one-time assembly success rate must be considered. Therefore, based on the existing research results, this paper analyzes and discusses the above three problems in detail, establishes a model of reducing the reference error of aeroengine rotor based on the measured data, and proposes a comprehensive prediction and optimization model based on the datum independent matrix, a fast algorithm for assembly phase is also illustrates.

This work is mainly focused on: eliminating the measurement datum errors to achieve the precise expression of the assembly features of the rotors at all levels, and establishing the global feature model based on the datum independent matrix to optimize the coaxiality of the front and rear fulcrum of the multi-stage rotor. Specifically: Section 2 shows how to eliminate the datum error and realize the representation of the geometric characteristics of the rotor itself with the datum independent matrix. Section 3 introduces the rotation matrix and datum independent matrix to establish the global feature model of the multi-stage rotor, illustrates the concept of coaxiality with the datum axis as the evaluation benchmark, the solution of the optimal assembly phase is also given. Section 4, experiments and data processing are carried out to verify the effectiveness of the proposed method. Section 5 summarizes the research and provides the study conclusions.

\section{Error Elimination Model of Measurement Datum}

Assembly features are regions of a part that are important for assembly purposes, they are made during fabrication, so they are, or corresponding to, fabrication features. Fabrication features are the regions of a part that are of importance for the purposes of creating the general shape of the part [3]. And due to the different processing methods and the limitation of the processing level, each size of the parts cannot reach the ideal value, and the processing surface of each part contains processing errors. As far as aeroengine rotor 
is concerned, the spigot surface of its assembly joint often becomes the assembly benchmark, and the test benchmark is usually determined by the turntable, which leads to the inconsistency between the test data and the geometric characteristics of the rotor itself. This inconsistency will eventually result in the failure of assembly accuracy prediction and further affect the overall performance of aeroengine rotor. Therefore, the model of datum error reduction under the separation condition of assembly and measurement should be established.

\subsection{Theory of Transformation Matrix}

Considering the technical means of rotor graded manufacturing and stacking assembly, the central position and slight inclination of contact surface of rotors at all levels is often the focus of our attention, so a homogeneous transformation matrix is naturally introduced to characterize the transformation of assembly characteristics of each level of rotor.

The $4 \times 4$ matrix transformation permits representation of both the relative position of two objects and their relative orientation. It tells us how to express points or vectors or plane in one coordinate frame in terms of another coordinate frame, it is important to understand that the matrix represents the location and orientation of an entire coordinate. Figure 3 shows the function of the transform schematically [3]. And the mathematical form of the transform is,

$$
T=\left[\begin{array}{ll}
R & p \\
0^{T} & 1
\end{array}\right]
$$

In this equation, $\mathrm{p}$ is a $3 \times 1$ displacement vector indicating the position of the new frame relative to the old one, while $\mathrm{R}$ is a 3 x 3 rotation matrix indicating the orientation of the new frame relative to the old one. (Superscript $\mathrm{T}$ indicates a vector or matrix transpose. By convention, all vectors are assumed to be column vectors, so a transposed vector is a row vector.)

Matrix R can be generated a number of ways, one way is to rotate once about each coordinate axis, this will generate one elemental rotation matrix. Matrix $\mathrm{R}$ can then be created by multiplying the elemental matrices into one another. The elemental matrices, as discussed in this article, are,

$$
T_{r o t}(x, \theta)=\left[\begin{array}{cccc}
1 & 0 & 0 & 0 \\
0 & \cos \theta & -\sin \theta & 0 \\
0 & \sin \theta & \cos \theta & 0 \\
0 & 0 & 0 & 1
\end{array}\right] \text { (2) }
$$




$$
\begin{aligned}
T_{\operatorname{rot}(y, \alpha)} & =\left[\begin{array}{cccc}
\cos \alpha & 0 & \sin \alpha & 0 \\
0 & 1 & 0 & 0 \\
-\sin \alpha & 0 & \cos \alpha & 0 \\
0 & 0 & 0 & 1
\end{array}\right] \quad(3) \\
T_{\operatorname{rot}(z, \beta)} & =\left[\begin{array}{cccc}
\cos \beta & -\sin \beta & 0 & 0 \\
\sin \beta & \cos \beta & 0 & 0 \\
0 & 0 & 1 & 0 \\
0 & 0 & 0 & 1
\end{array}\right] \quad(4)
\end{aligned}
$$

and we can easily find that, for rotation matrices [3],

$$
R^{-1}=R^{T}(5)
$$

A transform that simply repositions a frame without reorienting it is,

$$
T_{\text {trans }}=\left[\begin{array}{cccc}
1 & 0 & 0 & p_{x} \\
0 & 1 & 0 & p_{y} \\
0 & 0 & 1 & p_{z} \\
0 & 0 & 0 & 1
\end{array}\right] \text { (6) }
$$

The order in which T's are multiplied is important, and different sequences will create different results. For example,

$$
u=T_{\operatorname{trans}\left(p_{x}, 0,0\right)} * T_{\operatorname{rot}\left(z, \frac{\pi}{2}\right)} * v(7)
$$

It shows that rotates vector $v$ into a new orientation $u$ by first rotating $90^{\circ}$ about the $\mathrm{z}$ axis in the frame in which $v$ is measured, then move $p_{x}$ along the $\mathrm{x}$ axis in the same frame, as Fig. 4.

\subsection{Datum Error and Its Elimination}

The precision turntable is widely used in the runout measurement of rotating parts due to its high measurement accuracy and clear measurement principle. As a typical cylindrical rotating workpiece, the aeroengine rotor's runout measurement is mostly based on the precision turntable. In the assembly process of the rotor, one of the main indicators is the coaxiality of the multi-stage rotor. Based on the ISO definition of coaxiality, in this article, since we mainly focus on the center position of the upper and lower bottom surfaces of the rotor, the coaxiality is extended to twice the distance from the center of the rotor assembly surface to the datum axis [32]. Therefore, determining the center of the rotor assembly surface and the datum axis of rotation is the key. Considering the eccentricity and machining error of the rotor during the measurement process, the datum axis does not coincide with the measurement reference axis. Therefore, this 
section proposes a method to eliminate the reference error, while establishing the concept of the datum axis and its determination method.

Many literatures and experiments show that in the assembly of aeroengine rotors, the measurement of coaxiality is usually based on the axis of the front and rear fulcrums, which is close to the axis from the first stage rotor to the last stage rotor. Combined with the actual assembly situation and the simplicity of calculation, the datum axis of the rotor in this article is defined as: A straight line passing through the center $\mathrm{O}_{0}$ of the bottom surface of the rotor and perpendicular to the bottom surface, as shown in Fig. 5.

Rotor parts are currently mostly manufactured by graded manufacturing and stacked assembly techniques. Due to the limitation of machining level, the manufacturing of important assembly surface of rotor is not ideal and the surface is also uneven, at the same time, in the process of rotor runout test, owing to the center of the rotor cannot completely coincide with the center of the turntable, and the measurement plane of rotor is also difficult to be completely parallel to the plane of the turntable, so the test data often cannot truly reflect the actual geometric characteristics of the rotor, that is, the datum error is produced. If the measurement process of a single-stage rotor contains the influence of datum errors, it will accumulate in each level of stacking operation, which will seriously affect the final assembly accuracy. Therefore, Eliminating the datum error is extremely important.

Now take the Fig. 5 to help explain how to eliminate the reference error. $O_{E}$ is the origin of the reference coordinate system, that is, the center of the turntable, $\mathrm{O}_{0}$ is the center of the lower bottom surface of the rotor, $\mathrm{O}_{1}$ is the center of the upper bottom surface of the rotor, $\mathrm{E}$ represents the reference surface, 0 represents the lower bottom surface, and 1 represents the upper bottom surface. What can be obtained from the test data is the transformation matrix from $\mathrm{E}$ to 0 is $T_{E-0}$, and the transformation matrix from $\mathrm{E}$ to 1 is $T_{E-1}$. But what we need is the transformation matrix $T_{0-1}$ who represents the transform from 0 to 1 , therefore, we introduce the inverse matrix of $T_{E-0}$,

$$
T_{0-1}=T_{E-0}^{-1} * T_{E-1}(8)
$$

Equation (8) is implemented on the algorithm that the center of the rotor is adjusted to the center of the turntable, and the bottom of the rotor is adjusted to the plane of the turntable. Thus, the transformation of $\mathrm{O}_{0}$ to $\mathrm{O}_{1}$ is established, and the distance from $\mathrm{O}_{1}$ to the actual axis of rotation is obtained, at the same time, we call $T_{E-0}^{-1}$ as error reduction matrix and $T_{0-1}$ as datum independent matrix. 


\subsection{Datum independent matrix}

In the sec 2.2 , we established a transformation matrix to eliminate the benchmark error, and now we further introduce assembly features into the transformation matrix. Rama presented a comprehensive review of discrete data inspection algorithms to date [33]. They stated that $L_{p}$-norm formulation formed the basis for most of the frequently used fitting algorithms. The $L_{p}$-norm formulation is defined as,

$$
L_{p}=\left[\frac{1}{n} \sum_{i=1}^{n}\left|e_{i}\right|^{p}\right]^{1 / p}(9)
$$

where $\mathrm{p}$ is a control variable, $\mathrm{N}$ is the number of data points used, and $e_{i}$ is the shortest distance between the $i$ th data point and the feature. The most popular case of the $L_{p}$-norm formulation is the least squares method (LSM), which is found when $\mathrm{p}=2$.

The definition of surface topography is generally determined by the wave distance $\lambda$, when $\lambda<1 \mathrm{~mm}$, it shows roughness; When $1 \mathrm{~mm}<\lambda<10 \mathrm{~mm}$, waviness; when $\lambda>10 \mathrm{~mm}$, shape error. Owing to the probe radius of the inductive sensor is about $2 \mathrm{~mm}$, it can't touch the deep wave trough, so the test data can't reflect the micro surface roughness, but only include the waviness and shape error. This feature is in line with our actual assembly situation, because the rotor assembly contact surface often uses the interference fit, which makes the micro scale peak with lower stiffness produce plastic deformation, and only retains the waviness and shape with higher stiffness.

Then fitting the data of radial runout and axial runout of the upper and lower assembly surfaces of the rotor based on the least square method to obtain the fitting circle center: $(d x, d y)$ and fitting plane: $A x+$ $B y+C z+D=0$. Among them, $(d x, d y)$ is eccentric coordinates, $d \theta x=-B / C$ is the rotation angle of the fitting plane relative to the reference plane around the $\mathrm{x}$ axis, and $d \theta y=A / C$ is the rotation angle of the fitting plane relative to the reference plane around the y axis, and the rotation direction follows the righthanded spiral rule [34,35].

So $T_{E-0}$ and $T_{E-1}$ can be easily derived from the formulas (2), (3), (4) and (7),

$$
T_{E-0}=\left[\begin{array}{cccc}
1 & 0 & d_{\theta y 0} & d_{x 0} \\
0 & 1 & -d_{\theta x 0} & d_{y 0} \\
-d_{\theta y 0} & d_{\theta x 0} & 1 & 0 \\
0 & 0 & 0 & 1
\end{array}\right]
$$




$$
T_{E-1}=\left[\begin{array}{cccc}
1 & 0 & d_{\theta y 1} & d_{x 1} \\
0 & 1 & -d_{\theta x 1} & d_{y 1} \\
-d_{\theta y 1} & d_{\theta x 1} & 1 & z_{1} \\
0 & 0 & 0 & 1
\end{array}\right] \text { (11) }
$$

One thing to note is that, owing to $d_{\theta x} 、 d_{\theta y}$ are all smaller values. Without affecting the final accuracy, here we use the concept of equivalent infinity to replace $\sin d_{\theta x}$ with $d_{\theta x}$, and $\cos d_{\theta y}$ with 1 .

In the same way, each element of the datum independent matrix as Eq. (8) can be obtained.

\section{Stacking Model Based on Datum Independent Matrix}

As described in the sec 2.3 , the geometric characteristics of single stage rotor can be accurately characterized, and then the single stage rotor is stacked to form a complete aeroengine rotor. Therefore, it is necessary to establish an overall expression for accurate prediction and optimization.

\subsection{Global Characteristic Matrix}

Since the coaxiality is often used as one of the indexes to estimate the assembly accuracy of the rotor when assembling multi-stage rotors of aeroengines, in order to ensure good positioning and sufficiently small coaxiality value, the assembly position of the rotor often adopts a spigot structure, which has better centering and functionally restrained.

The motion of a rigid body can be described by six parameters, three related to linear motion and three related to rotation. Such a body is said to have six degrees of freedom. In this study, taking the two-stage rotor assembly as an example, due to the restriction of the spigot structure, we can clearly deduce that there is only one relative degree of freedom of rotation around the $\mathrm{z}$ axis between them, and the relative movement and rotation in other directions restricted. Owing to the manufacturing error of the rotor assembly surface, it is not an ideal plane. Therefore, the actual assembly surface obtained by fitting the measured data has a certain inclination angle relative to the ideal plane. As a result of the existence of this inclination angle, it can be rotated the rotor a certain angle to change the distance from the center of the rotor assembly surface to the datum axis, that is, to optimize the coaxiality, as shown in the Fig. 6.

Therefore, in order to establish a global transformation matrix of assembly features, we introduce the assembly feature rotation matrix of the assembly joint surface of the rotor to express the relative rotation 
angle $\theta_{z 1}$ between two adjacent rotors, where, $\theta_{z 1} \in[0,2 \pi]$, the assembly feature rotation matrix is defined as follows,

$$
T_{1-1^{\prime}}=\left[\begin{array}{cccc}
\cos \theta_{z 1} & -\sin \theta_{z 1} & 0 & 0 \\
\sin \theta_{z 1} & \cos \theta_{z 1} & 0 & 0 \\
0 & 0 & 1 & 0 \\
0 & 0 & 0 & 1
\end{array}\right](12)
$$

At this point, we can establish a transformation matrix $T_{0-2}$ for the two-stage rotor assembly which based on the datum independent matrix,

$$
T_{0-2}=T_{0-1} * T_{1-1} * T_{1^{\prime}-2}(13)
$$

In the same way, the global transformation matrix when the n-stage rotor participates in the assembly is,

$$
T_{0-n}=T_{0-1} * T_{1-1}, * T_{1^{\prime}-2} * \ldots \ldots * T_{m-m^{\prime}} * T_{m^{\prime}-n}(14)
$$

Among them, $\mathrm{m}=\mathrm{n}-1$, the composition of $T_{i-i+1}$ and $T_{i+1^{-}-i+2}$ is similar to Eq. (8).

The structure of $T_{0-n}$ is as follows,

$$
T_{0-n}=\left[\begin{array}{cc}
R_{x y z} & p_{x y z} \\
0^{T} & 1
\end{array}\right](15)
$$

Among them, $R_{x y z}$ represents the rotation of the bottom surface of the first stage rotor to the top surface of the top rotor around $\mathrm{x}, \mathrm{y}, \mathrm{z} ; p_{x y z}=\left[\begin{array}{lll}d_{x n} & d_{y n} & z_{n}\end{array}\right]^{T}$ represents translation along the $\mathrm{x}, \mathrm{y}$, and $\mathrm{z}$ directions.

\subsection{Solution of optimal assembly phase}

Based on the global characteristic matrix established in Section 3.1, now the expression can be established from the definition of the aforementioned coaxiality,

$$
\text { coaxiality }=2 *\left[\left(d_{x n}\right)^{2}+\left(d_{y n}\right)^{2}\right]^{1 / 2}(16)
$$

From the foregoing analysis and matrix operation, it can be seen that coaxiality is a function of $\theta_{z}$, so when coaxiality takes the minimum value, the corresponding assembly phase $\theta_{z}$ is the optimal assembly phase. In order to solve the problem simply, formula (17) is taken as the optimization function, the problem of optimal assembly phase is transformed into the problem of minimum trigonometric function of $\theta_{z}$.

$$
\mathrm{f}\left(\theta_{z}\right)=\left(d_{x n}\right)^{2}+\left(d_{y n}\right)^{2}(17)
$$

According to formula $(14,16)$, take the two-stage rotor assembly as an example, that is, when $n=2$ and $i=1, \theta_{z 1}$ is the adjustment phase of the lower-stage rotor relative to the upper-stage rotor. The direction is 
determined by the right hand. If the upper-stage rotor needs to be adjusted during assembly, the direction of rotation should take the reverse direction; on this basis, and then optimize 3, 4 up to $n$ level.

\subsection{Efficiency comparison with genetic algorithm}

As for the application of genetic algorithm in the field of rotor assembly optimization, we know its basic principle and have carried out the research on this method. The objective of optimization is to achieve the minimum coaxiality, that is, to make the center of all levels of rotor distributed around a reference axis as much as possible, in short, we usually find the optimal value by exhaustive method, the basic principle is dependent on the following analysis.

Assuming that two-stage rotors participate in assembly, there are 36 bolt holes in the circumference of the rotor, that is, 36 assembly phases can be selected, which are $10^{\circ}, 20^{\circ}, \ldots \ldots, 360^{\circ}$, the optimization principle of genetic algorithm is to calculate the coaxiality of all assembly phases and select the smallest one as the assembly phase, we can find that the amount of calculation is 36 . When n-stage rotors are involved in the assembly, there are 36 phases to choose between the first and second stages, and there are also 36 choices between the second and third stages, similarly, there are 36 choices between the $n-1$ and $n$ stage. At this time, the calculation amount becomes $36^{n-1}$, and the genetic algorithm is to select the assembly phase with the minimum coaxiality from the $36^{n-1}$ calculation results. In this method, the expression of coaxiality of nstage rotor is taken as the optimization function, and only the overall minimum is achieved, without considering the problem of intermediate stage, which is easy to produce the situation as shown in the Fig. 7.

The method we proposed is to put the assembly phase as a variable in the expression of coaxiality, and directly calculate the phase corresponding to the minimum value. In this way, the calculation amount of twostage rotor assembly is only 2 (one is similar to the matrix multiplication in genetic algorithm, and the other is to get the minimum value of function), When the n-stage rotor is involved in the assembly, the amount of calculation is only $2 \mathrm{n}$. Therefore, we believe that this method not only reduces the amount of calculation to improves the calculation efficiency, but also realizes the step-by-step optimization, and avoids the generation of curved rotor in the Fig. 7. It also has a good reference for the high-speed machinery stacked by multi-level parts. 


\section{Experimental validation}

In order to verify the effectiveness and reliability of the proposed datum error elimination method, the components were measured six times by using precision turntable, high precision inductance sensor and control software, the radial and face runout data of the upper and lower bottom surfaces of the rotor were collected in each measurement. MATLAB is used to filter and fit the six groups of data, and the values of rotor eccentricity and tilt caused by machining error and placement error are obtained, combined with matrix transformation and datum error elimination method, six groups of characteristic matrices are obtained through calculation, and their coaxiality is extracted as shown in the Table 1, the coaxiality distribution of six measurements is also made in Fig. 8. At the same time, we give the six measurement results without error elimination technology in Table 2 and Fig. 9, the results are based on the fact as Fig.10 show that only manufacturing errors are considered without considering test eccentricity.

The results show that: in the measurement and evaluation process of rotor coaxiality, although each measurement data is different, since it is impossible to ensure that each rotor is placed in the same position and reach the ideal value, but after the datum error elimination method proposed in this paper is used for data processing, the accuracy of the evaluation of rotor coaxiality, which represents the nature of the rotor itself, is about $93.82 \%$, that is to say, relative to the average value, the error of each processing result does not exceed $1.6 \mu \mathrm{m}$; also we can see that the coaxiality results without error elimination technology show low accuracy and extremely dispersity.

We can draw a conclusion: the coaxiality evaluation method after benchmark error reduction shows that the single result is closer to the mean value, so it is more reliable; however, the evaluation results obtained by the traditional method show great dispersion, and the single result can not reflect the geometric characteristics of the rotor itself.

When the coaxiality of a single-stage rotor is measured and evaluated accurately. Now we take the threestage rotor assembly as an example, combined with the actual measured axial runout and radial runout data of the aircraft rotor simulation test piece; use filtering, fitting technology and the stacking theory we proposed in this article to analyze the stacking optimization process of the three-stage rotor in detail. 
First, the single-stage rotor runout data, plane fitting and circle fitting based on the least square method, combined with the transformation matrix theory, use MATLAB to calculate the datum independent matrix of each stage of the rotor after eliminating the reference error.

Next, the stacking optimization of the two-stage rotor is carried out, and the rotation matrix $T_{1-1}$, is introduced between the first and second-stage rotors. It can be obtained by calculation that,

$$
\mathrm{f}\left(\theta_{z 1}\right)=-1.24 E^{-4} * \sin \left(\theta_{z 1}\right)+6.37 E^{-5} * \cos \left(\theta_{z 1}\right)+3.02 E^{-4}(18)
$$

The minimum point is $110^{\circ}$, That is, the optimal phase of the first and second rotor assembly is $\theta_{z 1}=$ $117^{\circ}$, and the coaxiality a $2=0.0255 \mathrm{~mm}$.

Substitute $\theta_{z 1}=110^{\circ}$ into Eq. (12), (13) to get $T_{0-2}$.

Finally, introduce $T_{2-2}$, between the two-stage rotor and the third-stage rotor, and calculate,

$$
\mathrm{f}\left(\theta_{z 2}\right)=1.52 E^{-4} * \sin \left(\theta_{z 2}\right)-7.64 E^{-5} * \cos \left(\theta_{z 2}\right)+2.35 E^{-4}(19)
$$

Get $\theta_{z 2}=297^{\circ}$, and $\mathrm{a} 3=0.0161 \mathrm{~mm}$.

Considering the limitation of the number and distribution of the circumferential bolt holes in the rotor flange, the optimal value calculated is often inconsistent with the angle of the bolt holes. Moreover, in engineering, it is more necessary to obtain an optimization area to meet the actual operation and demand than an optimal value. Therefore, we calculate the coaxiality in the range of $15^{\circ}$ degrees left and right expansion of the optimal assembly phase, and find that the coaxiality only has a very small change. The specific calculation value is that the coaxiality change caused by any assembly phase in the range of the assembly area does not exceed $0.001 \mathrm{~mm}$ relative to its minimum value. Therefore, the assembly within the optimization area can not only ensure the optimization effect, but also meet the actual engineering needs, and bring convenience to the specific operation and selection.

At the same time, in order to facilitate intuitive analysis, here is a comparison of the effects of $0^{\circ}$ phase assembly and the above optimized assembly, as shown in the Fig. 11.

Among them, the $0^{\circ}$ phase assembly coaxiality is calculated as: $\mathrm{b} 2=0.0382 \mathrm{~mm}, \mathrm{~b} 3=0.0252 \mathrm{~mm}$. Figure 11 can clearly show that when the assembly optimization method proposed in this paper is adopted, the coaxiality of the two-stage and three-stage rotors is significantly reduced compared with $0^{\circ}$ phase assembly, that is, the multi-stage rotor realizes "linear assembly" and assembly accuracy is also improved. 


\section{Summary and Conclusion}

In order to solve the inconsistency of test data caused by the separation condition of aeroengine rotor test and assembly, and the low efficiency of optimal assembly phase calculation, the datum error elimination and fast solution method of optimal assembly phase under this condition are studied. The main conclusions are as follows:

1. The datum error is eliminated and the datum independent matrix is established to reduce the information uncertainty in multiple assembly processes;

2. A prediction and optimization model based on datum independent matrix is established to improve the assembly accuracy and calculation efficiency, the optimal assembly phase range is also given.

3. The experimental results show that the method proposed in this paper can evaluate the geometric characteristics of the rotor, also realize the fast calculation of the optimal assembly phase, and reduce the coaxiality of the front and rear fulcrum of the multi-stage rotor.

\section{Funding}

This work is financially supported by the National Natural Science Foundation of China (No. 51875081).

\section{Author information}

\section{Affiliations}

School of Mechanical Engineering, Dalian University of Technology, No. 2, Linggong Road, Ganjingzi

District, Dalian, Liaoning Province, China

\section{Corresponding author}

Correspondence to Qingchao Sun

Correspondence to Xiaokai Mu

\section{Contributions}

Minghua Li established the error elimination and assembly model, he also drafted the manuscript. Yunlong Wang carried out relevant experiments and data processing, Qingchao Sun and Xiaokai Mu made suggestions and reviewed the manuscript. 


\section{Availability of data and material and code}

Not applicable.

\section{Ethics approval}

Not applicable.

\section{Consent to participate}

Not applicable.

\section{Consent for publication}

Not applicable.

\section{Conflict of interest}

The authors declare no competing interests.

\section{Reference}

[1] Zhu B, Yu NJ, Meng XH, Cheng YB, Ding SY, Shan FP (2015) Study on the tolerance analysis of aircraft engine assembly dimension chain. Journal of Test and Measurement Technology 29(02):177-184.

[2] Chen J, Li Y, Yu JF (2014) An effective tolerance allocation method for sheet metal assemblies. Manufacturing Automation 36(16):97-99.

[3] Whitney DE (2004) Mechanical assemblies. Oxford University Press, New York.

[4] Cai M, Yang JX, Wu ZT (2004) Mathematical model of cylindrical form tolerance. Journal of Zhejiang University Science 5(7):890-895. https://doi.org/10.1631/jzus.2004.0890

[5] Liu J, Wu FY, Wang J (2014) Optimization Technique of Aeroengine Rotor Assembly. Aero Engine 40 (03):75-78.

[6] Ju YP, Wu FY, Jin B, Wei XP, Zu JG (2018) Multi-stage disc rotor structure assembly process based on rotor runout and initial unbalance optimization. Aero Engine 44(06):83-90.

[7] Sun SM, Li Y (2020) Research on the influence of aero-engine rotor installation edge manufacturing error on assembly performance. Modern Manufacturing Technology and Equipment 56(07):126-131.

[8] Rao SS, Wu W (2005) Optimum tolerance allocation in mechanical assemblies using an interval method. 
Engineering Optimization 37(3):237-257. https://doi.org/10.1080/0305215512331328240

[9] Ding Y, Ceglarek D, Shi J (2000) Modeling and diagnosis of multistage manufacturing processes: Part I state space model. Japan/USA Symposium of Flexible Automation:1-8

[10] Thimm G, Britton GA, Cheong FS (2001) Controlling tolerance stacks for efficient manufacturing. The International Journal of Advanced Manufacturing Technology 18(1): 44-48.

[11] Zheng C, Jin S, Lai XM, Yu KG (2011) Assembly tolerance allocation using a coalitional game method. Engineering Optimization 43(7):763-778.

[12] Griffin P, Ballinger IA, Jaekle DE, Jackson A (2003) Design and manufacturing of a lightweight fuel tank assembly. 39th AIAA/ASME/SAE/ASEE Joint Propulsion Conference and Exhibit, Huntsville, Alabama, July 20-23, 2003, AIAA: 2003-4606. https://doi.org/10.2514/6.2003-4606

[13] Kulyk M, Lastivka I, Tereshchenko Y (2012) Effect of hysteresis in axial compressors of gas turbine engines. Aviation 16(4):97-102. https://doi.org/10.3846/16487788.2012.753679

[14] Klocke F, Veselovac D, Auerbach T, Seidner R (2008) Intelligent Assembly for Aero Engine Components. 1st International Conference Intelligent Robotics and Applications, Wuhan, PEOPLES R CHINA, OCT 15-17, 2008, 5315: 927-935. https://doi.org/10.1007/978-3-540-88518-4_99

[15] Yang Z, Hussain T, Popov AA, Mcwillam S (2011) A Comparison of Different Optimization Techniques for Variation Propagation Control in Mechanical Assembly. IOP Conference Series: Materials Science and Engineering 26(1):012017. https://doi.org/10.1088/1757-899x/26/1/012017

[16] Yang Z, McWilliam S, Popov AA, Hussain T (2013) A probabilistic approach to variation propagation control for straight build in mechanical assembly. The International Journal of Advanced Manufacturing Technology 64(5-8):1029-1047. https://doi.org/10.1007/s00170-012-4071-x

[17] Yang Z, Hussain T, Popov AA, Mcwillam S (2011) Novel optimization technique for variation propagation control in an aero-engine assembly. Proceedings of the Institution of Mechanical Engineers, Part B: Journal of Engineering Manufacture 225(1):100-111. https://doi.org/10.1243/09544054JEM2043

[18] Yang Z, McWilliam S, Popov AA, Hussain T (2013) Dimensional variation propagation analysis in straight-build mechanical assemblies using a probabilistic approach. Journal of Manufacturing Systems 32(2):348-356. https://doi.org/10.1016/j.jmsy.2012.11.008

[19] Meng XH, Shan FP (2016) Assembling quality prediction of aero-engine rotor parts. Manufacturing 
Automation 38(05):61-65.

[20] Shan FP, Li ZM, Zhu B (2015) Modeling and analysis of assembly deviation of typical aero-engine rotor parts. Manufacturing Automation 37(07):100-103.

[21] Ai YY, Zhou HL, Sun D, Wang Z, Zhang FL, Tian J (2015) Vibration analysis and control of aero-engine. Journal of Shenyang University of Aeronautics and Astronautics 32(05):1-25.

[22] Jin S, Ding SY, Li ZM, Yang FY, Ma X (2018) Point-based solution using Jacobian-Torsor theory into partial parallel chains for revolving components assembly. Journal of Manufacture System 46:46-58. https://doi.org/10.1016/j.jmsy.2017.11.003

[23] Ding SY, Jin S, Li ZM, Wei ZQ, Yang FY (2018) Deviation Propagation Model and Optimization of Concentricity for Aero-engine Rotor Assembly. Journal of Shanghai Jiao tong University 52(01):54-62.

[24] Ding SY, Zheng XH (2020) Rotor assembly accuracy control method based on improved Jacobian-Torsor theory. Journal of Aeronautics:1-16.

[25] Chen H, Jin S, Li ZM, Lai XM (2014) A comprehensive study of three-dimensional tolerance analysis methods. Computer-Aided Design 53:1-13. https://doi.org/10.1016/j.cad.2014.02.014

[26] Chen ZY, Liu Y, Zhou P, Liu GT (2018) Assembly tolerance analysis based on improved Taguchi method. Computer Integrated Manufacturing Systems 24(05):1200-1206.

[27] Chen ZY, Liu Y, Zhou P, Liu GT (2018) Assembly Success Rate Calculation Method for Aero-Engine Based on Improved Taguchi Method. Journal of Propulsion Technology 39(3):653-659.

[28] Sun HB, Yan JX, Wei XH (2020) Chang Z. Aero-engine assembly technology driven by digital twin. China Mechanical Engineering 31(07):833-841.

[29] Asante JN (2009) A small displacement torsor model for tolerance analysis in a workpiece-fixture assembly. Proceedings of the Institution of Mechanical Engineers, Part B, Journal of Engineering Manufacture 223(8):1005-1020. https://doi.org/10.1243/09544054JEM1337

[30] Li H, Zhu HP, Li PG, He F (2014) Tolerance analysis of mechanical assemblies based on small displacement torsor and deviation propagation theories. International Journal of Advanced Manufacture Technology 72(1-4):89-99. https://doi.org/10.1007/s00170-014-5630-0

[31] Khan HA, Butt SU, Baqai AA, Saeed HA (2018) A new approach to predict the flexibility and precision of manufacturing systems using geometric constraints and small displacement torsors. Procedia 
Manufacturing 17:294-301. https://doi.org/10.1016/j.promfg.2018.10.049

[32] Sun CZ (2017) Research on the measurement method of multi-stage rotor coaxiality based on vector projection. Harbin Institute of Technology.

[33] Rama H, Turek S, Rajmohan S, Anand S (2011) A comprehensive methodology for runout tolerance evaluation using discrete data. International Journal of Advanced Manufacturing Technology 56(58):663-676. https://doi.org/10.1007/s00170-011-3218-5

[34] Mu XK, Sun QC, Sun W, Wang YL, Wang CH, Wang XB (2018) 3D Tolerance modeling and geometric precision analysis of plane features for flexible parts. Engineering Computations 35(7):2557-2576. https://doi.org/10.1108/EC-10-2017-0388

[35] Sun QC, Zhao BB, Liu X, Mu XK, Zhang YL (2019) Assembling deviation estimation based on the real mating status of assembly. Computer-Aided Design:244-255. https://doi.org/10.1016/j.cad.2019.06.001 


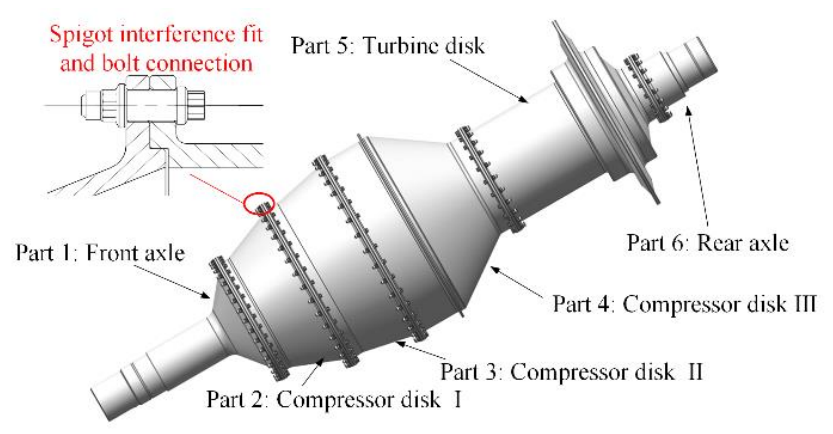

Fig. 1 The high pressure compressor simulator of aeroengine rotor
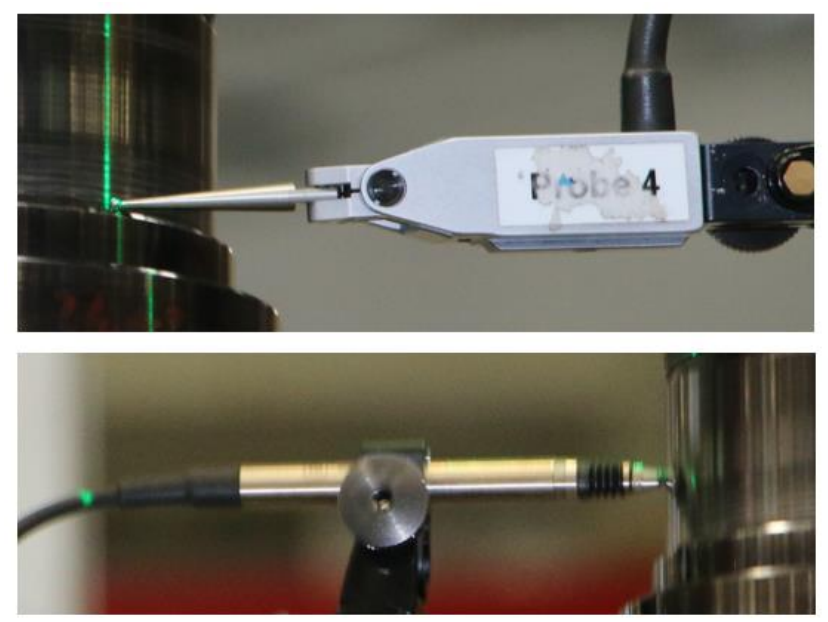

Fig. 2 Radial and axial run out test

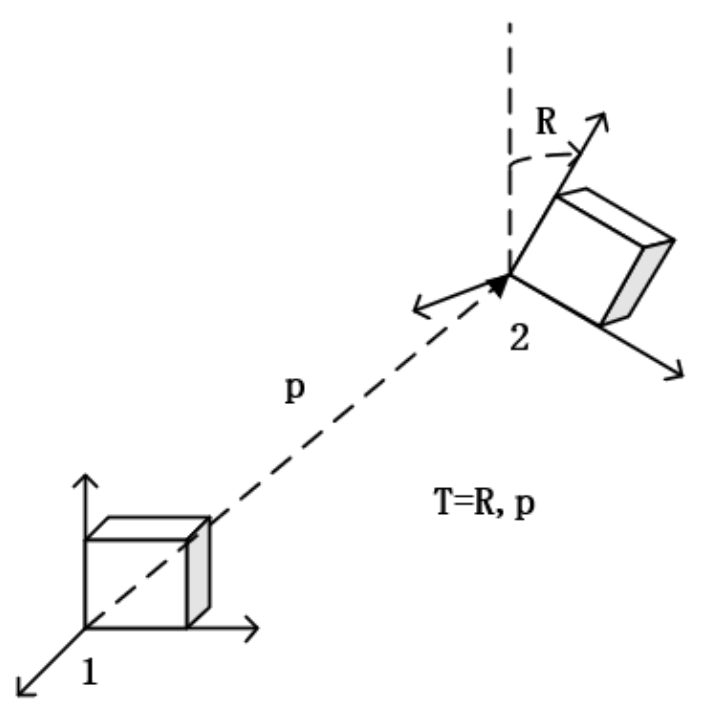

Fig. 3 Schematic Representation of a Transform 


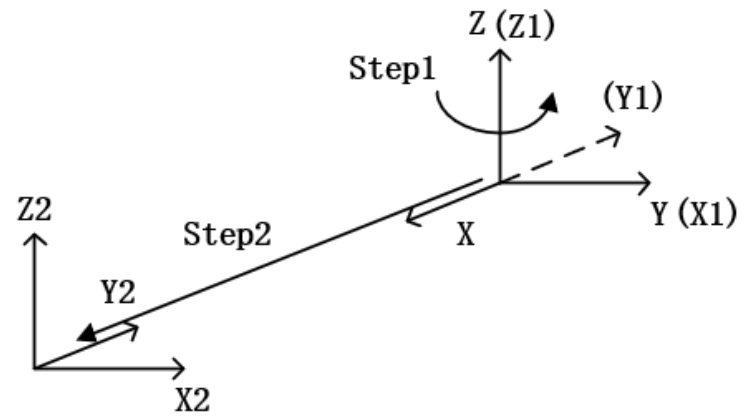

Fig. 4 Illustration of Interpreting Equation (7)

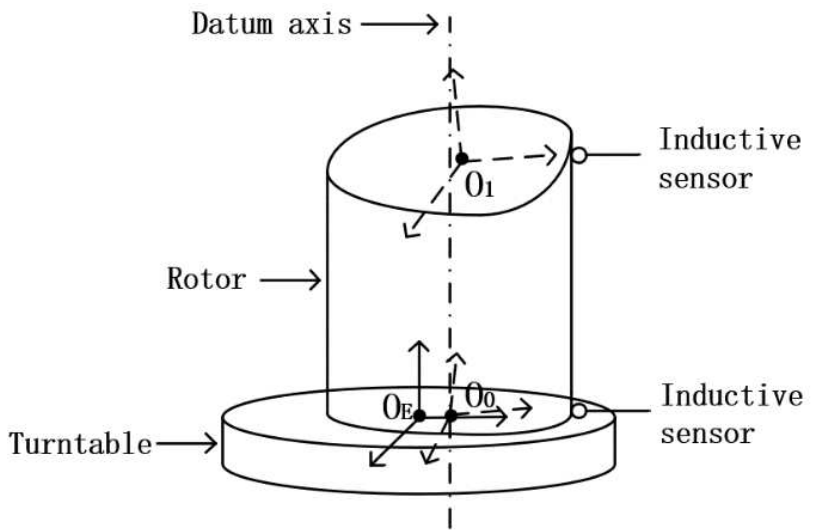

Fig. 5 Test principle considering manufacturing error and placement eccentricity

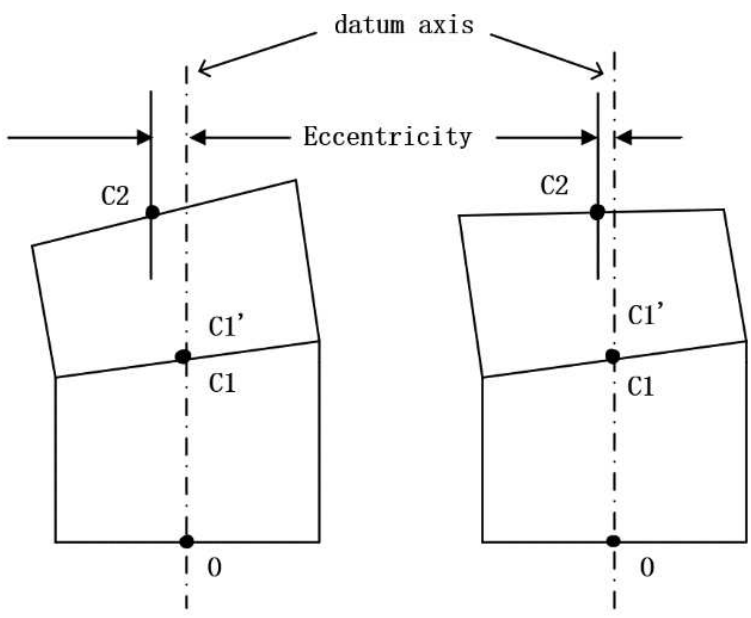

Fig. 6 Schematic diagram of two-stage rotor optimized assembly 


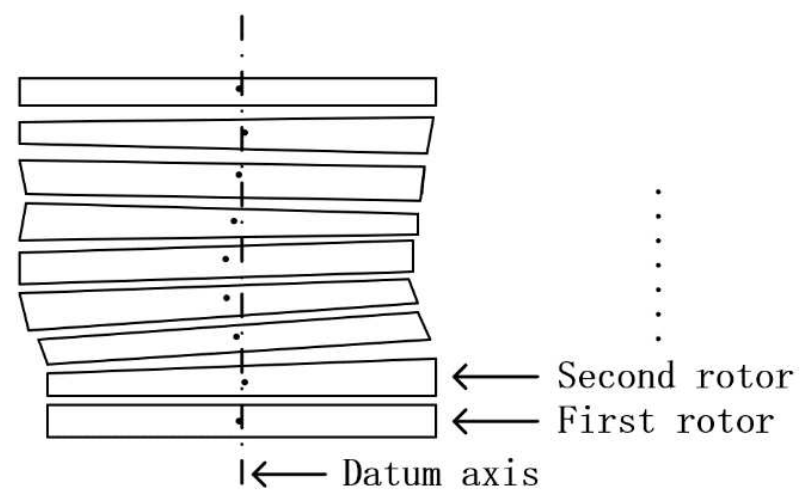

Fig. 7 Schematic diagram of curved rotor

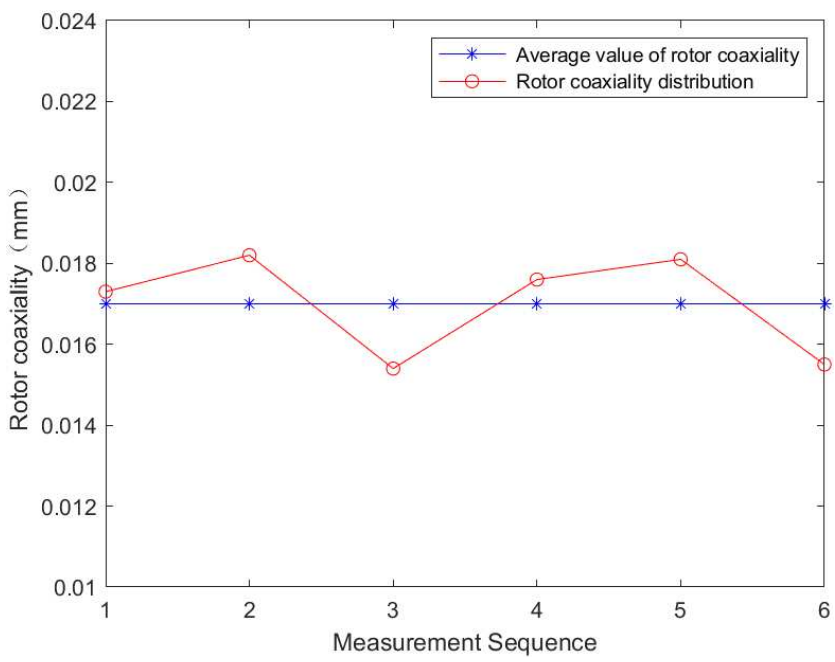

Fig. 8 Rotor coaxiality distribution based on error elimination technique

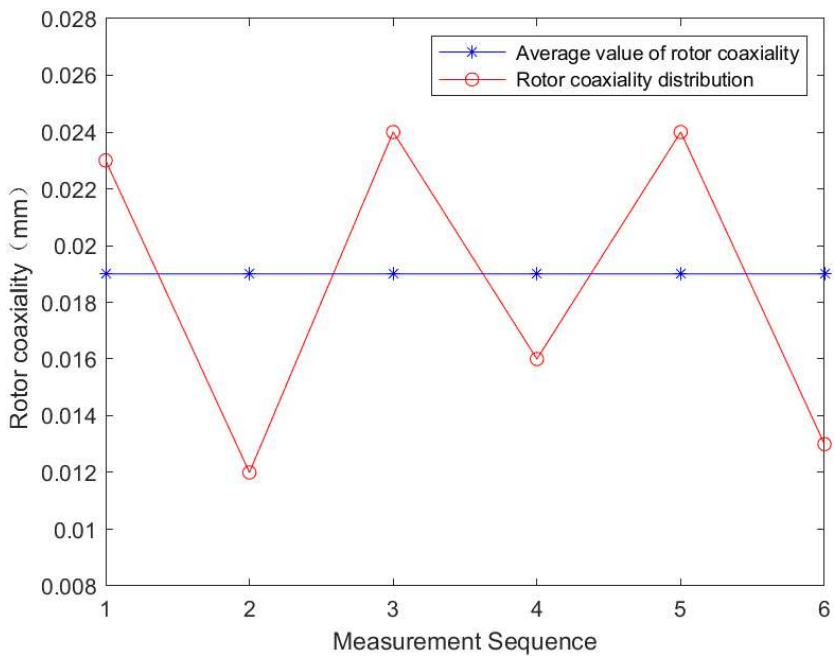

Fig. 9 Rotor coaxiality distribution without error elimination 


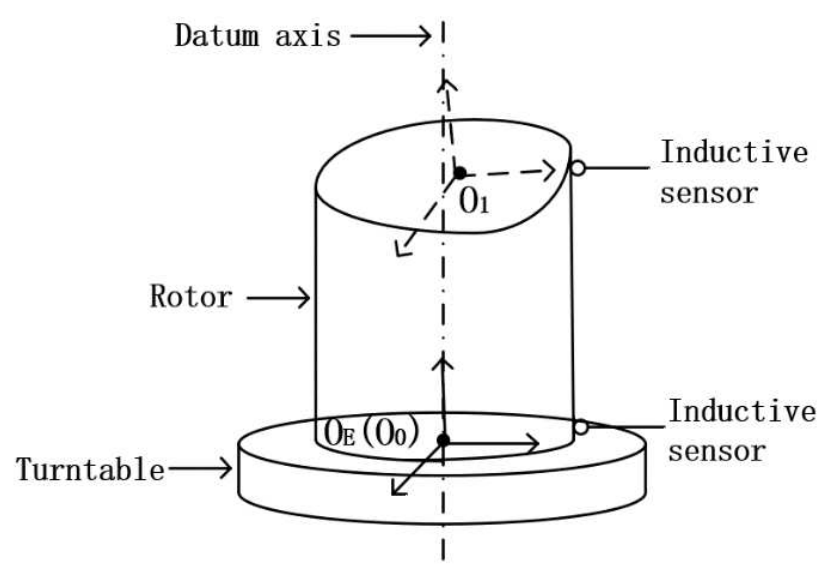

Fig. 10 Test principle only considering manufacturing error

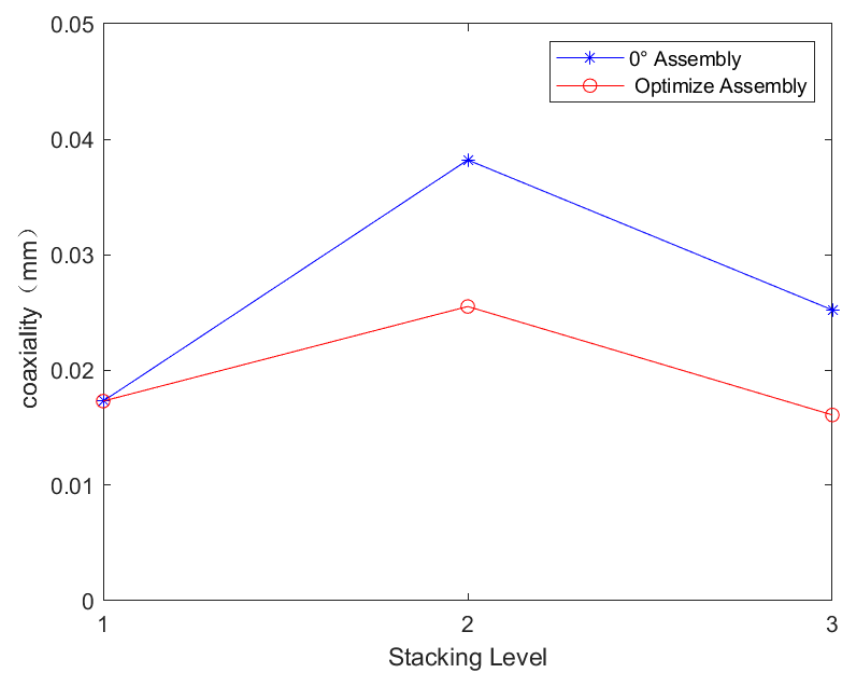

Fig. 11 Coaxiality comparison between random assembly and optimized assembly 
Table 1 Results of rotor coaxiality based on datum independent matrix

\begin{tabular}{ccc}
\hline Measurement sequence & Rotor coaxiality $(\mathrm{mm})$ & Measurement accuracy \\
\hline 1 & 0.0154 & $90.59 \%$ \\
2 & 0.0176 & $96.47 \%$ \\
3 & 0.0182 & $92.94 \%$ \\
4 & 0.0173 & $98.24 \%$ \\
5 & 0.0155 & $91.18 \%$ \\
6 & 0.0181 & $93.53 \%$ \\
\hline average value & 0.0170 & $93.82 \%$ \\
\hline
\end{tabular}

Table 2 Results of rotor coaxiality without error elimination

\begin{tabular}{ccc}
\hline Measurement sequence & Rotor coaxiality $(\mathrm{mm})$ & Measurement accuracy \\
\hline 1 & 0.0131 & $68.42 \%$ \\
2 & 0.0241 & $73.68 \%$ \\
3 & 0.0232 & $78.95 \%$ \\
4 & 0.0124 & $63.16 \%$ \\
5 & 0.0163 & $84.21 \%$ \\
6 & 0.0241 & $73.68 \%$ \\
\hline average value & 0.0192 & $73.66 \%$ \\
\hline
\end{tabular}

